\title{
Theoretical study of the spin competition in small-sized Al clusters
}

\author{
Omar López-Estrada and Emilio Orgaz* \\ Departamento de Física y Química Teórica, Facultad de Química, Universidad Nacional \\ Autónoma de México, Cd. Universitaria, CP 04510, México, D.F. México \\ E-mail: emilio.orgaz@gmail.com
}




\section{SUPPORTING INFORMATION}

\section{AL RESULTS}

TABLE I. Total electronic energy for the $\mathrm{Al}$ atom (in a.u.) in the doublet (D) spin state at different levels of theory ${ }^{\mathrm{a}}$.

\begin{tabular}{ccccc}
\hline \hline & PBE & PW91 & TPSS & -0.9502 \\
\hline SDecp & -0.9411 & -0.9437 & -0.3625 & -0.9499 \\
D95 & -0.2097 & -0.3271 & -0.3397 & -0.2642 \\
DGDZVP & -0.1906 & -0.3087 & -0.3654 & -0.2396 \\
DGTZVP & -0.2156 & -0.3337 & -0.3777 & -0.2645 \\
TZVP & -0.2245 & -0.3416 & -0.3798 & -0.2789 \\
Def2TZVPP & -0.2276 & -0.3452 & -0.3767 & -0.2782 \\
\hline aug-cc-pVDZ & -0.2233 & -0.3402 & -0.3828 & -0.2841 \\
aug-cc-pVTZ & -0.2296 & -0.3467 & -0.3858 & -0.2870 \\
aug-cc-pVQZ & -0.2333 & -0.3507 & -0.3890 & -0.2898 \\
CBS & -0.2373 & -0.3551 & & \\
\hline \hline
\end{tabular}

a In order to simplify the numeric values is required to add -242 a.u. except por the SDecp computation where is required to add -1 a.u.

b The CBS extrapolation is computed for the aug-cc-pVxZ basis set $(x=D, T, Q)$.

\section{$\mathrm{AL}_{2}$ RESULTS}

TABLE II. $\mathrm{Al}_{2}$ total electronic energy (in a.u.) for the ${ }^{1} \Sigma_{g}^{-}$and ${ }^{3} \Sigma_{g}^{-},{ }^{3} \Pi_{u}$ spin states at different levels of theory ${ }^{\mathrm{a}}$.

\begin{tabular}{|c|c|c|c|c|c|c|}
\hline & \multicolumn{3}{|c|}{$\mathrm{PBE}$} & \multicolumn{3}{|c|}{ PW91 } \\
\hline & ${ }^{1} \Sigma_{g}^{-}$ & ${ }^{3} \Sigma_{g}^{-}$ & ${ }^{3} \Pi_{u}$ & ${ }^{1} \Sigma_{g}^{-}$ & ${ }^{3} \Sigma_{g}^{-}$ & ${ }^{3} \Pi_{u}$ \\
\hline SDecp & -0.8976 & -0.9352 & -0.9375 & & -0.9404 & -0.9429 \\
\hline D95 & -0.4483 & -0.4723 & -0.4743 & -0.6836 & -0.7075 & -0.7095 \\
\hline DGDZVP & -0.4140 & -0.4400 & -0.4391 & -0.6505 & -0.6763 & -0.6756 \\
\hline DGTZVP & -0.4643 & -0.4906 & -0.4894 & -0.7009 & -0.7269 & -0.7259 \\
\hline TZVP & -0.4829 & -0.5090 & -0.5080 & -0.7175 & -0.7433 & -0.7425 \\
\hline Def2TZVPP & -0.4902 & -0.5170 & -0.5154 & -0.7257 & -0.7522 & -0.7509 \\
\hline aug-cc-pVDZ & -0.4675 & -0.5065 & -0.5057 & -0.7007 & -0.7405 & -0.7400 \\
\hline aug-cc-pVTZ & -0.4939 & -0.5206 & -0.5194 & -0.7286 & -0.7550 & -0.7540 \\
\hline aug-cc-pVQZ & -0.4869 & -0.5277 & -0.5263 & -0.7361 & -0.7627 & -0.7616 \\
\hline \multirow[t]{3}{*}{ CBS } & -0.4739 & -0.5350 & -0.5336 & -0.7420 & -0.7708 & -0.7696 \\
\hline & & TPSS & & & revTPSS & \\
\hline & ${ }^{1} \Sigma_{g}^{-}$ & ${ }^{3} \Sigma_{g}^{-}$ & ${ }^{3} \Pi_{u}$ & ${ }^{1} \Sigma_{g}^{-}$ & ${ }^{3} \Sigma_{g}^{-}$ & ${ }^{3} \Pi_{u}$ \\
\hline SDecp & -0.9238 & -0.9371 & -0.9423 & -0.9188 & -0.9315 & -0.9373 \\
\hline D95 & -0.7499 & -0.7724 & -0.7755 & -0.5524 & -0.5740 & -0.5769 \\
\hline DGDZVP & -0.7088 & -0.7343 & -0.7341 & -0.5147 & -0.5329 & -0.5325 \\
\hline DGTZVP & -0.7602 & -0.7860 & -0.7855 & -0.5581 & -0.5831 & -0.5824 \\
\hline TZVP & -0.7175 & -0.8105 & -0.8104 & -0.5868 & -0.6115 & -0.6112 \\
\hline Def2TZVPP & -0.7906 & -0.8169 & -0.8161 & -0.5916 & -0.6171 & -0.6161 \\
\hline aug-cc-pVDZ & -0.7826 & -0.8090 & -0.8090 & -0.5851 & -0.6106 & -0.6105 \\
\hline aug-cc-pVTZ & -0.7962 & -0.8225 & -0.8220 & -0.5984 & -0.6238 & -0.6232 \\
\hline aug-cc-pVQZ & -0.8019 & -0.8284 & -0.8278 & -0.6035 & -0.6291 & -0.6283 \\
\hline CBS & -0.8074 & -0.8344 & -0.8335 & -0.6083 & -0.6343 & -0.6333 \\
\hline
\end{tabular}

${ }^{a}$ In order to simplify the numeric values is required to add -484 a.u. except por the SDecp computation where is required to add -3 a.u. 
TABLE III. $\mathrm{Al}_{2}$ total energy (in a.u.) for the singlet ${ }^{1} \Sigma_{g}^{-}$and triplet ${ }^{3} \Sigma_{g}^{-},{ }^{3} \Pi_{u}$ spin states obtained with aug-cc-pVxZ $\left(\mathrm{x}=\mathrm{D}, \mathrm{T}, \mathrm{Q}\right.$.) basis sets and the complete basis set extrapolation (CBS) using perturbative MP4 and CCSD(T) methods ${ }^{\mathrm{a}}$, and energy difference $\Delta={ }^{3} \Pi_{u}-{ }^{3} \Sigma_{g}^{-}$(in eV/atom).

\begin{tabular}{|c|c|c|c|c|c|c|}
\hline & & TZVP & aug-cc-pVDZ & aug-cc-pVTZ & aug-cc-pVQZ & CBS \\
\hline \multirow{4}{*}{ MP4 } & ${ }^{1} \Sigma_{g}^{-}$ & -0.8555 & -0.8729 & -0.8973 & -0.9033 & -0.9077 \\
\hline & ${ }^{3} \Sigma_{g}^{-}$ & -0.8779 & -0.8853 & -0.9093 & -0.9154 & -0.9200 \\
\hline & ${ }^{3} \Pi_{u}$ & -0.8794 & -0.8877 & -0.9111 & -0.9167 & -0.9207 \\
\hline & $\Delta$ & -0.020 & -0.068 & -0.048 & -0.035 & -0.020 \\
\hline \multirow{4}{*}{$\operatorname{CCSD}(\mathrm{T})$} & ${ }^{1} \Sigma_{g}^{-}$ & -0.8603 & -0.8782 & -0.9025 & -0.9086 & -0.9132 \\
\hline & ${ }^{3} \Sigma_{g}^{-}$ & -0.8797 & -0.8874 & -0.9115 & -0.9178 & -0.9227 \\
\hline & ${ }^{3} \Pi_{u}$ & -0.8821 & -0.8903 & -0.9137 & -0.9196 & -0.9240 \\
\hline & $\Delta$ & -0.032 & -0.081 & -0.061 & -0.048 & -0.037 \\
\hline
\end{tabular}

${ }^{\text {a }}$ In order to simplify the numeric values is required to add -483 a.u.

TABLE IV. $\mathrm{Al}_{2}$ difference in the total electronic energy between the ${ }^{3} \Pi_{u}$ and the ${ }^{3} \Sigma_{g}^{-}$electronic states $\left({ }^{3} \Pi_{u}-{ }^{3} \Sigma_{g}^{-}\right.$in eV/atom $)$ at differents levels of theory. A positive value indicates that the ${ }^{3} \Sigma_{g}^{-}$state is the more stable structure.

\begin{tabular}{ccccc}
\hline \hline & PBE & PW91 & TPSS & revTPSS \\
\hline SDecp & -0.031 & -0.034 & -0.071 & -0.079 \\
D95 & -0.027 & -0.027 & -0.042 & 0.039 \\
DGDZVP & 0.012 & 0.010 & 0.003 & 0.005 \\
DGTZVP & 0.016 & 0.014 & 0.007 & 0.002 \\
TZVP & 0.014 & 0.011 & 0.011 & 0.005 \\
Def2TZVPP & 0.022 & 0.018 & 0.000 & 0.014 \\
\hline aug-cc-pVDZ & 0.011 & 0.007 & 0.007 & 0.008 \\
aug-cc-pVTZ & 0.016 & 0.014 & 0.008 & 0.011 \\
aug-cc-pVQZ & 0.019 & 0.015 & 0.012 \\
CBS & 0.019 & 0.016 & 0.014 \\
\hline \hline
\end{tabular}

a The CBS extrapolation is computed for the aug-cc-pVxZ basis set $(x=D, T, Q)$.

TABLE V. $\mathrm{Al}_{2}$ bond length $\mathrm{BL}$ (in $\AA$ ) and vibrational frequency $\nu$ (in $\mathrm{cm}^{-1}$ ) at different levels of theory. ${ }^{\mathrm{a}}$

\begin{tabular}{|c|c|c|c|c|c|c|c|c|c|}
\hline & & \multicolumn{2}{|c|}{$\mathrm{PBE}$} & \multicolumn{2}{|c|}{ PW91 } & \multicolumn{2}{|c|}{ TPSS } & \multicolumn{2}{|c|}{ revTPSS } \\
\hline & & BL & $\nu$ & $\mathrm{BL}$ & $\nu$ & BL & $\nu$ & $\mathrm{BL}$ & $\nu$ \\
\hline \multirow{9}{*}{${ }^{3} \Sigma_{g}^{-}$} & SDD & 2.57 & 312.6 & 2.57 & 310.9 & 2.59 & 294.5 & 2.61 & $\overline{282.4}$ \\
\hline & D95 & 2.57 & 298.3 & 2.57 & 298.5 & 2.57 & 295.9 & 2.57 & 295.0 \\
\hline & DGDZVP & 2.50 & 338.9 & 2.50 & 337.5 & 2.48 & 347.0 & 2.48 & 346.8 \\
\hline & DGTZVP & 2.49 & 340.1 & 2.49 & 338.8 & 2.48 & 347.5 & 2.48 & 347.7 \\
\hline & TZVP & 2.50 & 342.8 & 2.49 & 341.3 & 2.49 & 349.8 & 2.49 & 349.0 \\
\hline & Def2TZVPP & 2.49 & 344.0 & 2.49 & 342.9 & 2.48 & 349.9 & 2.48 & 349.6 \\
\hline & AUG-cc-pVDZ & 2.51 & 340.4 & 2.50 & 338.7 & 2.50 & 346.2 & 2.50 & 345.4 \\
\hline & AUG-cc-pVTZ & 2.49 & 343.0 & 2.49 & 341.9 & 2.48 & 348.9 & 2.49 & 348.2 \\
\hline & AUG-cc-pVQZ & 2.49 & 342.1 & 2.49 & 341.1 & 2.48 & 349.0 & 2.48 & 348.5 \\
\hline \multirow{9}{*}{${ }^{3} \Pi_{u}$} & SDD & 2.86 & 239.4 & 2.86 & 239.3 & 2.90 & 218.8 & 2.92 & 210.3 \\
\hline & D95 & 2.88 & 218.4 & 2.88 & 218.9 & 2.87 & 221.9 & 2.87 & 221.5 \\
\hline & DGDZVP & 2.77 & 255.6 & 2.77 & 255.6 & 2.75 & 262.6 & 2.75 & 263.5 \\
\hline & DGTZVP & 2.77 & 254.1 & 2.77 & 254.4 & 2.75 & 259.8 & 2.75 & 260.9 \\
\hline & TZVP & 2.76 & 258.5 & 2.76 & 258.9 & 2.75 & 262.5 & 2.75 & 262.9 \\
\hline & Def2TZVPP & 2.76 & 260.3 & 2.75 & 260.8 & 2.74 & 265.3 & 2.74 & 265.8 \\
\hline & AUG-cc-pVDZ & 2.77 & 258.4 & 2.77 & 258.5 & 2.75 & 262.9 & 2.75 & 263.4 \\
\hline & AUG-cc-pVTZ & 2.76 & 259.4 & 2.75 & 259.8 & 2.74 & 263.9 & 2.74 & 264.8 \\
\hline & AUG-cc-pVQZ & 2.76 & 258.7 & 2.75 & 259.0 & 2.74 & 264.2 & 2.74 & 264.9 \\
\hline
\end{tabular}

${ }^{\mathrm{a}}$ Experimental data ${ }^{1}{ }^{3} \Sigma_{g}^{-} \mathrm{d}=2.4665 \pm 0.0024 \AA$ and $\nu=350.01 \mathrm{~cm}^{-1},{ }^{3} \Pi_{u} \mathrm{~d}=2.7011 \pm 0.0015 \AA, \nu=285.8 \mathrm{~cm}^{-1}$ 
TABLE VI. $\mathrm{Al}_{2}$ bond length $\mathrm{BL}$ (in $\AA$ ) and vibrational frequency $\nu$ (in $\mathrm{cm}^{-1}$ ) at MP4 and $\mathrm{CCSD}(\mathrm{T})$ levels of theory ${ }^{\mathrm{a}}$

\begin{tabular}{|c|c|c|c|c|c|}
\hline & & \multicolumn{2}{|c|}{ MP4 } & \multicolumn{2}{|c|}{$\operatorname{CCSD}(\mathrm{T})$} \\
\hline & & $\mathrm{BL}$ & $\nu$ & BL & $\nu$ \\
\hline \multirow{4}{*}{${ }^{3} \Sigma_{g}^{-}$} & TZVP & 2.487 & 355.0 & 2.493 & 348.6 \\
\hline & aug-cc-pVDZ & 2.524 & 343.0 & 2.538 & 330.5 \\
\hline & aug-cc-pVTZ & 2.485 & 357.3 & 2.497 & 345.1 \\
\hline & aug-cc-pVQZ & 2.473 & 361.7 & 2.485 & 348.2 \\
\hline \multirow{4}{*}{${ }^{3} \Pi_{u}$} & TZVP & 2.711 & 292.8 & 2.714 & 289.8 \\
\hline & aug-cc-pVDZ & 2.763 & 274.7 & 2.767 & 271.8 \\
\hline & aug-cc-pVTZ & 2.725 & 284.4 & 2.728 & 282.1 \\
\hline & aug-cc-pVQZ & 2.714 & 286.6 & 2.717 & 284.2 \\
\hline
\end{tabular}

${ }^{\mathrm{a}}$ Experimental data ${ }^{1}{ }^{3} \Sigma_{g}^{-} \mathrm{d}=2.4665 \pm 0.0024 \AA$ and $\nu=350.01 \mathrm{~cm}^{-1},{ }^{3} \Pi_{u} \mathrm{~d}=2.7011 \pm 0.0015 \AA, \nu=285.8 \mathrm{~cm}^{-1}$

\section{$\mathrm{AL}_{3}$ RESULTS}

TABLE VII. $\mathrm{Al}_{3}$ total electronic energy (in a.u.) for the doublet ${ }^{2} A_{1}^{\prime}$ spin state in $\mathrm{D}_{3 h}$ symmetry at different levels of theory ${ }^{\mathrm{a}}$.

\begin{tabular}{lcccc}
\hline \hline & PBE & PW91 & TPSS & revTPSS \\
\hline SDecp & -0.9520 & -0.9597 & -0.9495 & -0.9403 \\
D95 & -0.7529 & -1.1059 & -1.2031 & -0.9069 \\
DGDZVP & -0.7177 & -1.0721 & -1.1606 & -0.8604 \\
DGTZVP & -0.7939 & -1.1484 & -1.2379 & -0.9356 \\
TZVP & -0.8225 & -1.1741 & -1.2755 & -0.9787 \\
Def2TZVPP & -0.8351 & -1.1882 & -1.2856 & -0.9876 \\
\hline aug-cc-pVDZ & -0.8168 & -1.1678 & -1.2713 & -0.9755 \\
aug-cc-pVTZ & -0.8401 & -1.1919 & -1.2935 & -0.9971 \\
aug-cc-pVQZ & -0.8505 & -1.2032 & -1.3022 & -1.0049 \\
CBS & -0.8608 & -1.2147 & -1.3105 & -1.0121 \\
\hline \hline
\end{tabular}

${ }^{\text {a }}$ In order to simplify the numeric values is required to add -726 a.u. except por the SDecp computation where is required to add -5 a.u.

TABLE VIII. $\mathrm{Al}_{3}$ difference in the total electronic energy between the doublet ${ }^{2} A_{1}^{\prime}$ (D) and the quadruplet ${ }^{4} A_{2}(\mathrm{Q})$ spin states (D-Q in eV/atom) at different levels of theory. A negative value indicates that the doublet spin state is the more stable structure.

\begin{tabular}{ccccc}
\hline \hline & PBE & PW91 & TPSS & revTPSS \\
\hline SDecp & -0.012 & -0.011 & 0.021 & 0.028 \\
D95 & -0.001 & -0.001 & 0.001 & -0.008 \\
DGDZVP & -0.066 & -0.065 & -0.065 & -0.072 \\
DGTZVP & -0.069 & -0.069 & -0.065 & -0.073 \\
TZVP & -0.073 & -0.073 & -0.070 & -0.076 \\
Def2TZVPP & -0.072 & -0.072 & -0.067 & -0.073 \\
\hline aug-cc-pVDZ & -0.054 & -0.054 & -0.050 & -0.057 \\
aug-cc-pVTZ & -0.067 & -0.067 & -0.062 & -0.067 \\
aug-cc-pVQZ & -0.068 & -0.069 & -0.063 & -0.069 \\
CBS & -0.068 & -0.069 & -0.064 & -0.070 \\
\hline \hline
\end{tabular}

a The CBS extrapolation is computed for the aug-cc-pVxZ basis set $(x=D, T, Q)$. 
TABLE IX. $\mathrm{Al}_{3}$ bond length (BL in $\AA$ ), frequencies $\left(\nu\right.$ in $\left.\mathrm{cm}^{-1}\right)$ and vibrational symmetries for the doublet spin state at $\mathrm{D}_{3 h}$ symmetry for different functional and basis sets. ${ }^{a}$

\begin{tabular}{|c|c|c|c|c|c|c|}
\hline & \multicolumn{3}{|c|}{$\mathrm{PBE}$} & \multicolumn{3}{|c|}{ PW91 } \\
\hline & $\mathrm{BL}_{1,2,3}$ & $\nu_{1} \mathrm{e}^{\prime}$ & $\nu_{2} \mathrm{a}_{1}^{\prime}$ & $\mathrm{BL}_{1,2,3}$ & $\nu_{1} \mathrm{e}^{\prime}$ & $\nu_{2} \mathrm{a}_{1}^{\prime}$ \\
\hline SDecp & 2.6109 & 202.3425 & 322.1159 & 2.6112 & 201.8167 & 320.2566 \\
\hline D95 & 2.6117 & 196.7139 & 292.4224 & 2.6093 & 196.2349 & 292.6797 \\
\hline DGDZVP & 2.5300 & 235.2321 & 359.8775 & 2.5295 & 234.2888 & 358.2569 \\
\hline DGTZVP & 2.5242 & 237.3618 & 360.3572 & 2.5229 & 236.7903 & 359.2189 \\
\hline TZVP & 2.5243 & 239.4787 & 363.4228 & 2.5230 & 238.7745 & 361.9902 \\
\hline Def2TZVPP & 2.5195 & 242.1622 & 362.3698 & 2.5177 & 241.5804 & 361.2367 \\
\hline aug-cc-pVDZ & 2.5436 & 234.1704 & 353.9313 & 2.5428 & 233.3984 & 352.2805 \\
\hline aug-cc-pVTZ & 2.5248 & 240.8338 & 360.6088 & 2.5231 & 240.3785 & 359.5554 \\
\hline \multirow[t]{3}{*}{ aug-cc-pVQZ } & 2.5200 & 241.0874 & 360.2354 & 2.5177 & 240.7388 & 359.3859 \\
\hline & \multicolumn{3}{|c|}{ TPSS } & \multicolumn{3}{|c|}{ revTPSS } \\
\hline & $\overline{B L}_{1,2,3}$ & $\nu_{1} \mathrm{e}^{\prime}$ & $\nu_{2} \mathrm{a}_{1}^{\prime}$ & $\overline{B L}_{1,2,3}$ & $\nu_{1} \mathrm{e}^{\prime}$ & $\nu_{2} \mathrm{a}_{1}^{\prime}$ \\
\hline SDecp & 2.6271 & 187.9201 & 309.2057 & 2.6376 & 183.2245 & 303.7382 \\
\hline D95 & 2.6096 & 190.9166 & 290.8647 & 2.6072 & 191.2918 & 292.3834 \\
\hline DGDZVP & 2.5155 & 236.295 & 368.8238 & 2.5140 & 237.6421 & 369.8634 \\
\hline DGTZVP & 2.5126 & 237.4138 & 367.7554 & 2.5112 & 238.1355 & 368.5182 \\
\hline TZVP & 2.5157 & 238.6849 & 369.1275 & 2.5140 & 239.175 & 369.7850 \\
\hline Def2TZVPP & 2.5110 & 242.2057 & 368.1033 & 2.5091 & 242.3719 & 368.8246 \\
\hline aug-cc-pVDZ & 2.5354 & 232.9805 & 358.9799 & 2.5332 & 234.3683 & 360.5695 \\
\hline aug-cc-pVTZ & 2.5174 & 240.5467 & 365.3656 & 2.5162 & 240.3634 & 365.5279 \\
\hline aug-cc-pVQZ & 2.5107 & 241.9420 & 366.7693 & 2.5099 & 241.7301 & 366.8882 \\
\hline
\end{tabular}

a Experimental frequencies: stretching $a_{1}^{\prime} 357 \pm 10 \mathrm{~cm}^{-1}$, bending mode $e^{\prime} 240 \pm 10 \mathrm{~cm}^{-1},^{2}$

TABLE X. $\mathrm{Al}_{3}$ total energy (in a.u.) for the doublet ${ }^{2} A_{1}^{\prime}$ spin state obtained with aug-cc-pVxZ ( $\mathrm{x}=\mathrm{D}, \mathrm{T}, \mathrm{Q}$.) basis sets and the complete basis set extrapolation (CBS) using perturbative $\operatorname{CCSD}(\mathrm{T})$ and MP4 methods ${ }^{\mathrm{a}}$.

\begin{tabular}{lcr}
\hline \hline & CCSD(T) & MP4 \\
\hline TZVP & -0.8770 & -0.8757 \\
aug-cc-pVDZ & -0.8825 & -0.8827 \\
aug-cc-pVTZ & -0.9274 & -0.9263 \\
aug-cc-pVQZ & -0.9392 & -0.9382 \\
CBS & -0.9483 & -0.9477 \\
\hline \hline
\end{tabular}

${ }^{\text {a }}$ In order to simplify the numeric values is required to add -725 a.u.

TABLE XI. $\mathrm{Al}_{3}$ bond length (BL in $\AA$ ), frequencies $\left(\nu\right.$ in $\left.\mathrm{cm}^{-1}\right)$ and vibrational symmetries for the doublet spin state in $\mathrm{D}_{3 h}$ symmetry at $\operatorname{CCSD}(\mathrm{T})$ and MP4 theory level and different basis sets. ${ }^{a}$

\begin{tabular}{|c|c|c|c|c|c|c|}
\hline & \multicolumn{3}{|c|}{$\operatorname{CCSD}(\mathrm{T})$} & \multicolumn{3}{|c|}{ MP4 } \\
\hline & $\mathrm{BL}_{1,2,3}$ & $\nu_{1} \mathrm{e}^{\prime}$ & $\nu_{2} \mathrm{a}_{1}^{\prime}$ & $\mathrm{BL}_{1,2,3}$ & $\nu_{1} \mathrm{e}^{\prime}$ & $\nu_{2} \mathrm{a}_{1}^{\prime}$ \\
\hline TZVP & 2.5396 & 240.2741 & 366.4829 & 2.6037 & 182.0016 & 335.6776 \\
\hline aug-cc-pVDZ & 2.5165 & 257.4809 & 393.2657 & 2.6721 & 240.4952 & 326.8684 \\
\hline aug-cc-pVTZ & 2.5493 & 232.1042 & 353.8035 & 2.6408 & 195.4111 & 313.5326 \\
\hline aug-cc-pVQZ & 2.5315 & 238.0173 & 359.8348 & 2.6205 & 191.0311 & 303.2244 \\
\hline
\end{tabular}

${ }^{\text {a }}$ Experimental frequencies: stretching $a_{1}^{\prime} 357 \pm 10 \mathrm{~cm}^{-1}$, bending mode $e^{\prime} 240 \pm 10 \mathrm{~cm}^{-1},^{2}$ 
TABLE XII. $\mathrm{Al}_{3}^{+}$vertical ionization energy (in eV) from the neutral doublet spin ground state at $\mathrm{D}_{3 h}$ symmetry to the ${ }^{1} A_{2}^{\prime \prime}$ and to the ${ }^{3} A_{1}^{\prime}$ states, at different levels of theory. The experimental value has been reported to be $6.46 \pm 0.04 \mathrm{eV}^{3}$.

\begin{tabular}{|c|c|c|c|c|c|c|c|c|}
\hline & \multicolumn{2}{|c|}{$\mathrm{PBE}$} & \multicolumn{2}{|c|}{ PW91 } & \multicolumn{2}{|c|}{ TPSS } & \multicolumn{2}{|c|}{ revTPSS } \\
\hline & ${ }^{1} A_{1}^{\prime}$ & ${ }^{3} A_{2}^{\prime \prime}$ & ${ }^{1} A_{1}^{\prime}$ & ${ }^{3} A_{2}^{\prime \prime}$ & ${ }^{1} A_{1}^{\prime}$ & ${ }^{3} A_{2}^{\prime \prime}$ & ${ }^{1} A_{1}^{\prime}$ & ${ }^{3} A_{2}^{\prime \prime}$ \\
\hline SDecp & 7.57 & 6.53 & 7.59 & 6.56 & 6.81 & 6.44 & 6.77 & 6.41 \\
\hline D95 & 6.89 & 6.65 & 6.91 & 6.68 & 6.91 & 6.71 & 6.92 & 6.75 \\
\hline DGDZVP & 6.78 & 6.58 & 6.82 & 6.62 & 6.82 & 6.63 & 6.83 & 6.66 \\
\hline DGTZVP & 6.78 & 6.58 & 6.82 & 6.62 & 6.81 & 6.63 & 6.83 & 6.66 \\
\hline TZVP & 6.81 & 6.60 & 6.84 & 6.62 & 6.82 & 6.62 & 6.83 & 6.65 \\
\hline Def2TZVPP & 6.77 & 6.55 & 6.81 & 6.58 & 6.79 & 6.57 & 6.80 & 6.60 \\
\hline aug-cc-pVDZ & 6.78 & 6.52 & 6.81 & 6.55 & 6.79 & 6.54 & 6.80 & 6.57 \\
\hline aug-cc-pVTZ & 6.77 & 6.54 & 6.81 & 6.57 & 6.78 & 6.56 & 6.79 & 6.58 \\
\hline aug-cc-pVQZ & 6.76 & 6.54 & 6.80 & 6.57 & 6.79 & 6.57 & 6.80 & 6.59 \\
\hline
\end{tabular}




\section{$\mathrm{AL}_{4}$ RESULTS}

TABLE XIII. $\mathrm{Al}_{4}$ total electronic energy (in a.u.) for the singlet (S) and the triplet (T) spin states at different levels of theory ${ }^{\mathrm{a}}$.

\begin{tabular}{|c|c|c|c|c|c|c|c|c|}
\hline & \multicolumn{2}{|c|}{$\mathrm{PBE}$} & \multicolumn{2}{|c|}{ PW91 } & \multicolumn{2}{|c|}{ TPSS } & \multicolumn{2}{|c|}{ revTPSS } \\
\hline & S & $\mathrm{T}$ & S & $\mathrm{T}$ & $\mathrm{S}$ & $\mathrm{T}$ & $\mathrm{S}$ & $\mathrm{T}$ \\
\hline SDecp & -0.9644 & -0.9742 & -0.9747 & -0.9848 & -0.9582 & -0.9700 & -0.9450 & -0.9565 \\
\hline D95 & -1.0359 & -1.0466 & -1.5067 & -1.5174 & -1.6356 & -1.6413 & -1.2403 & -1.2462 \\
\hline DGDZVP & -0.9827 & -0.9909 & -1.4551 & -1.4633 & -1.5728 & -1.5597 & -1.1726 & -1.1594 \\
\hline DGTZVP & -1.0841 & -1.0923 & -1.5566 & -1.5648 & -1.6757 & -1.6622 & -1.2726 & -1.2591 \\
\hline TZVP & -1.1220 & -1.1303 & -1.5907 & -1.5990 & -1.7253 & -1.7335 & -1.3298 & -1.3379 \\
\hline Def2TZVPP & -1.1385 & -1.1475 & -1.6090 & -1.6181 & -1.7387 & -1.7476 & -1.3416 & -1.3504 \\
\hline aug-cc-pVDZ & -1.1147 & -1.1238 & -1.5826 & -1.5917 & -1.7206 & -1.7294 & -1.3262 & -1.3121 \\
\hline aug-cc-pVTZ & -1.1454 & -1.1546 & -1.6143 & -1.6234 & -1.7493 & -1.7585 & -1.3542 & -1.3633 \\
\hline aug-cc-pVQZ & -1.1587 & -1.1679 & -1.6288 & -1.6381 & -1.7606 & -1.7697 & -1.3643 & -1.3733 \\
\hline CBS & -1.1718 & -1.1811 & -1.6436 & -1.6528 & -1.7714 & -1.7803 & -1.3737 & -1.3791 \\
\hline
\end{tabular}

${ }^{\text {a }}$ In order to simplify the numeric values is required to add -968 a.u. except por the SDecp computation where is required to add -7 a.u.

TABLE XIV. $\mathrm{Al}_{4}$ difference in the total electronic energy between the singlet $(\mathrm{S})$ and the triplet $(\mathrm{T})$ spin states $(\mathrm{T}-\mathrm{S}$ in $\mathrm{eV}$ /atom) at different levels of theory. A negative value indicates that the triplet spin state is the more stable structure.

\begin{tabular}{ccccc}
\hline \hline & PBE & PW91 & TPSS & -0.080 \\
\hline SDecp & -0.067 & -0.068 & -0.039 & -0.078 \\
D95 & -0.073 & -0.073 & 0.090 & -0.040 \\
DGDZVP & -0.056 & -0.056 & 0.092 & 0.090 \\
DGTZVP & -0.056 & -0.055 & -0.056 & -0.092 \\
TZVP & -0.057 & -0.057 & -0.061 & -0.055 \\
Def2TZVPP & -0.061 & -0.061 & -0.060 & 0.096 \\
aug-cc-pVDZ & -0.062 & -0.062 & -0.062 & -0.062 \\
aug-cc-pVTZ & -0.063 & -0.062 & -0.062 & -0.061 \\
aug-cc-pVQZ & -0.063 & -0.063 & -0.061 & -0.037 \\
CBS & -0.063 & -0.063 & & \\
\hline \hline
\end{tabular}

TABLE XV. $\mathrm{Al}_{4} \mathrm{BL}$ and angle $(\theta)$ at different levels of theory.

\begin{tabular}{cccccccc}
\hline \hline & \multicolumn{2}{c}{ PBE } & \multicolumn{2}{c}{ PW91 } & \multicolumn{2}{c}{ TPSS } & \multicolumn{2}{c}{ revTPSS } \\
& BL & $\theta$ & BL & $\theta$ & BL & $\theta$ & \multicolumn{1}{c}{ BL } \\
\hline SDecp & 2.723 & 81.093 & 2.724 & 81.499 & 2.723 & 78.864 & 2.728 \\
D95 & 2.755 & 83.321 & 2.754 & 83.730 & 2.666 & 71.561 & 2.663 \\
DGDZVP & 2.572 & 68.227 & 2.572 & 68.419 & 2.6165 & 63.9555 & 2.6165 \\
DGTZVP & 2.568 & 68.127 & 2.567 & 68.215 & 2.619 & 63.8675 & 2.619 \\
TZVP & 2.567 & 67.898 & 2.566 & 68.140 & 2.559 & 67.606 & 2.556 \\
Def2TZVPP & 2.563 & 68.178 & 2.561 & 68.334 & 2.554 & 67.805 & 2.552 \\
aug-cc-pVDZ & 2.582 & 68.487 & 2.581 & 68.632 & 2.573 & 68.114 & 2.6504 \\
aug-cc-pVTZ & 2.567 & 68.164 & 2.566 & 68.333 & 2.559 & 67.891 & 67.531 \\
aug-cc-pVQZ & 2.564 & 68.267 & 2.562 & 68.421 & 2.554 & 67.845 & 2.559 \\
\hline \hline
\end{tabular}




\section{AL $_{6}$ RESULTS}

TABLE XVI. $\mathrm{Al}_{6}$ total electronic energy (in a.u.) for the singlet (S) and the triplet (T) spin states at different levels of theory ${ }^{\mathrm{a}}$.

\begin{tabular}{|c|c|c|c|c|c|c|c|c|}
\hline & \multicolumn{2}{|c|}{$\mathrm{PBE}$} & \multicolumn{2}{|c|}{ PW91 } & \multicolumn{2}{|c|}{ TPSS } & \multicolumn{2}{|c|}{ revTPSS } \\
\hline & $\mathrm{S}$ & $\mathrm{T}$ & S & $\mathrm{T}$ & $\mathrm{S}$ & $\mathrm{T}$ & $\mathrm{S}$ & $\mathrm{T}$ \\
\hline SDecp & -0.0329 & -0.0357 & -0.0473 & -0.0505 & -0.0202 & -0.0230 & -0.0022 & -0.0043 \\
\hline D95 & -0.6457 & -0.6481 & -1.3507 & -1.3535 & -1.5437 & -1.5456 & -0.9550 & -0.9544 \\
\hline DGDZVP & -0.5787 & -0.5772 & -1.2859 & -1.2841 & -1.4641 & -1.4629 & -0.8671 & -0.8658 \\
\hline DGTZVP & -0.7310 & -0.7296 & -1.4384 & -1.4366 & -1.6186 & -1.6176 & -1.0173 & -1.0160 \\
\hline TZVP & -0.7880 & -0.7864 & -1.4898 & -1.4880 & -1.6931 & -1.6920 & -1.1029 & -1.1016 \\
\hline Def2TZVPP & -0.8170 & -0.8172 & -1.5213 & -1.5211 & -1.7174 & -1.7179 & -1.1248 & -1.1250 \\
\hline aug-cc-pVDZ & -0.7794 & -0.7788 & -1.4796 & -1.4786 & -1.6885 & -1.6882 & -1.1000 & -1.0994 \\
\hline aug-cc-pVTZ & -0.8267 & -0.8269 & -1.5285 & -1.5283 & -1.7328 & -1.7333 & -1.1433 & -1.1435 \\
\hline aug-cc-pVQZ & -0.8463 & -0.8465 & -1.5500 & -1.5499 & -1.7491 & -1.7496 & -1.1578 & -1.1580 \\
\hline $\mathrm{CBS}^{\mathrm{b}}$ & -0.8653 & -0.8655 & -1.5715 & -1.5713 & -1.7642 & -1.7647 & -1.1998 & -1.2017 \\
\hline
\end{tabular}

${ }^{a}$ In order to simplify the numeric values is required to add -1453 a.u. except for the SDecp computation where is required to add -12 a.u.

$\mathrm{b}$ The CBS extrapolation is computed for the aug-cc-pVxZ basis set $(\mathrm{x}=\mathrm{D}, \mathrm{T}, \mathrm{Q})$.

TABLE XVII. $\mathrm{Al}_{6}$ difference in the total electronic energy between the singlet $(\mathrm{S})$ and the triplet $(\mathrm{T})$ spin states $(\mathrm{S}-\mathrm{T}$ in $\mathrm{eV} /$ atom) at different levels of theory. A positive value indicates that the triplet spin state is the more stable structure.

\begin{tabular}{ccccc}
\hline \hline & PBE & PW91 & TPSS & revTPSS \\
\hline SDecp & 0.012 & 0.015 & 0.013 & 0.010 \\
D95 & 0.011 & 0.013 & 0.008 & -0.003 \\
DGDZVP & -0.007 & -0.008 & -0.005 & -0.006 \\
DGTZVP & -0.007 & -0.008 & -0.005 & -0.006 \\
TZVP & -0.007 & -0.009 & -0.005 & -0.006 \\
Def2TZVPP & 0.001 & -0.001 & 0.002 & 0.001 \\
\hline aug-cc-pVDZ & -0.003 & -0.005 & -0.001 & -0.003 \\
aug-cc-pVTZ & 0.001 & -0.001 & 0.002 & 0.001 \\
aug-cc-pVQZ & 0.001 & -0.001 & 0.002 & 0.001 \\
\hline \hline
\end{tabular}

TABLE XVIII. $\mathrm{Al}_{6}$ average nearest-neighbor $\langle d\rangle$ (in $\AA$ ) and dispersion $\sigma$ (in $\AA$ ) at different levels of theory.

\begin{tabular}{|c|c|c|c|c|c|c|c|c|}
\hline & \multicolumn{2}{|c|}{$\mathrm{PBE}$} & \multicolumn{2}{|c|}{ PW91 } & \multicolumn{2}{|c|}{ TPSS } & \multicolumn{2}{|c|}{ revTPSS } \\
\hline & $\langle d\rangle$ & $\sigma$ & $\langle d\rangle$ & $\sigma$ & $\langle d\rangle$ & $\sigma$ & $\langle d\rangle$ & $\sigma$ \\
\hline SDecp & 3.02 & 0.51 & 3.03 & 0.51 & 3.03 & 0.51 & 3.04 & $\overline{0.51}$ \\
\hline D95 & 3.06 & 0.51 & 3.06 & 0.51 & 3.06 & 0.51 & 3.10 & 0.57 \\
\hline DGDZVP & 2.77 & 0.20 & 2.78 & 0.20 & 2.76 & 0.20 & 2.75 & 0.19 \\
\hline DGTZVP & 2.77 & 0.20 & 2.77 & 0.20 & 2.76 & 0.20 & 2.76 & 0.20 \\
\hline TZVP & 2.76 & 0.20 & 2.76 & 0.20 & 2.75 & 0.19 & 2.75 & 0.19 \\
\hline Def2TZVPP & 2.73 & 0.10 & 2.76 & 0.20 & 2.72 & 0.10 & 2.72 & 0.10 \\
\hline aug-cc-pVDZ & 2.79 & 0.20 & 2.80 & 0.21 & 2.78 & 0.20 & 2.78 & 0.20 \\
\hline aug-cc-pVTZ & 2.74 & 0.10 & 2.77 & 0.20 & 2.76 & 0.20 & 2.73 & 0.10 \\
\hline aug-cc-pVQZ & 2.73 & 0.10 & 2.77 & 0.20 & 2.75 & 0.19 & 2.72 & 0.10 \\
\hline
\end{tabular}




\section{AL $_{8}$ RESULTS}

TABLE XIX. $\mathrm{Al}_{8}$ total electronic energy (in a.u.) for the singlet (S) and the triplet (T) spin states at different levels of theory ${ }^{\mathrm{a}}$.

\begin{tabular}{|c|c|c|c|c|c|c|c|c|}
\hline & \multicolumn{2}{|c|}{$\mathrm{PBE}$} & \multicolumn{2}{|c|}{ PW91 } & \multicolumn{2}{|c|}{ TPSS } & \multicolumn{2}{|c|}{ revTPSS } \\
\hline & $\mathrm{S}$ & $\mathrm{T}$ & S & $\mathrm{T}$ & $\mathrm{S}$ & $\mathrm{T}$ & $\mathrm{S}$ & $\mathrm{T}$ \\
\hline SDecp & -0.1138 & -0.1030 & -0.1330 & -0.1216 & -0.0950 & -0.0860 & -0.0717 & -0.0631 \\
\hline D95 & -0.2706 & -0.2598 & -1.2100 & -1.1989 & -1.4713 & -1.4606 & -0.6855 & -0.6748 \\
\hline DGDZVP & -0.1754 & -0.1658 & -1.1177 & -1.1071 & -1.3502 & -1.3535 & -0.5558 & -0.5595 \\
\hline DGTZVP & -0.3789 & -0.3695 & -1.3216 & -1.3134 & -1.5565 & -1.5601 & -0.7562 & -0.7601 \\
\hline TZVP & -0.4462 & -0.4453 & -1.3903 & -1.3796 & -1.6547 & -1.6585 & -0.8695 & -0.8734 \\
\hline Def2TZVPP & -0.4911 & -0.4842 & -1.4297 & -1.4212 & -1.6861 & -1.6904 & -0.8976 & -0.9021 \\
\hline aug-cc-pVDZ & -0.4398 & -0.4299 & -1.3730 & -1.3621 & -1.6556 & -1.6481 & -0.8637 & -0.8651 \\
\hline aug-cc-pVTZ & -0.5043 & -0.4972 & -1.4396 & -1.4302 & -1.7063 & -1.7098 & -0.9217 & -0.9253 \\
\hline aug-cc-pVQZ & -0.5298 & -0.5219 & -1.4678 & -1.4588 & -1.7279 & -1.7320 & -0.9410 & -0.9452 \\
\hline $\mathrm{CBS}^{\mathrm{b}}$ & -0.5543 & -0.5448 & -1.4957 & -1.4868 & -1.7491 & -1.7525 & -0.9581 & -0.9628 \\
\hline
\end{tabular}

${ }^{a}$ In order to simplify the numeric values is required to add -1938 a.u. except for the SDecp computation where is required to add -16 a.u.

b The CBS extrapolation is computed for the aug-cc-pVXZ basis set $(x=D, T, Q)$.

TABLE XX. $\mathrm{Al}_{8}$ difference in the total electronic energy between the singlet $(\mathrm{S})$ and the triplet $(\mathrm{T})$ spin states $(\mathrm{S}-\mathrm{T}$ in $\mathrm{eV} /$ atom) at different levels of theory. A positive value indicates that the triplet spin state is the more stable structure.

\begin{tabular}{ccccc}
\hline \hline & PBE & PW91 & TPSS & -0.031 \\
SDecp & -0.037 & -0.039 & -0.036 & -0.037 \\
D95 & -0.037 & -0.038 & 0.011 & 0.013 \\
DGDZVP & -0.033 & -0.036 & 0.012 & 0.013 \\
DGTZVP & -0.032 & -0.028 & 0.013 & 0.013 \\
TZVP & -0.003 & -0.036 & 0.015 & 0.015 \\
Def2TZVPP & -0.024 & -0.029 & -0.026 & 0.005 \\
\hline aug-cc-pVDZ & -0.034 & -0.037 & 0.012 & 0.012 \\
aug-cc-pVTZ & -0.024 & -0.032 & 0.014 & 0.014 \\
aug-cc-pVQZ & -0.027 & -0.031 & & \\
\hline \hline
\end{tabular}

TABLE XXI. $\mathrm{Al}_{8}$ average nearest-neighbor $\langle d\rangle$ (in $\AA$ ) and dispersion $\sigma$ (in $\AA$ ) at different levels of theory.

\begin{tabular}{|c|c|c|c|c|c|c|c|c|}
\hline & \multicolumn{2}{|c|}{ PBE } & \multicolumn{2}{|c|}{ PW91 } & \multicolumn{2}{|c|}{ TPSS } & \multicolumn{2}{|c|}{ revTPSS } \\
\hline & $\langle d\rangle$ & $\sigma$ & $\langle d\rangle$ & $\sigma$ & $\langle d\rangle$ & $\sigma$ & $\langle d\rangle$ & $\sigma$ \\
\hline SDecp & 3.00 & 0.49 & 3.01 & 0.48 & 3.01 & 0.49 & 3.01 & 0.50 \\
\hline D95 & 2.92 & 0.35 & 2.92 & 0.35 & 2.84 & 0.25 & 2.91 & 0.35 \\
\hline DGDZVP & 2.90 & 0.55 & 2.90 & 0.55 & 2.69 & 0.09 & 2.69 & 0.09 \\
\hline DGTZVP & 2.90 & 0.55 & 2.90 & 0.55 & 2.69 & 0.09 & 2.69 & 0.09 \\
\hline TZVP & 2.78 & 0.25 & 2.89 & 0.54 & 2.69 & 0.08 & 2.68 & 0.08 \\
\hline Def2TZVPP & 2.69 & 0.16 & 2.89 & 0.54 & 2.81 & 0.39 & 2.68 & 0.08 \\
\hline aug-cc-pVDZ & 2.92 & 0.55 & 2.92 & 0.55 & 2.90 & 0.55 & 2.71 & 0.09 \\
\hline aug-cc-pVTZ & 2.90 & 0.55 & 2.90 & 0.55 & 2.69 & 0.08 & 2.69 & 0.08 \\
\hline aug-cc-pVQZ & 2.89 & 0.54 & 2.89 & 0.54 & 2.69 & 0.08 & 2.68 & 0.08 \\
\hline
\end{tabular}



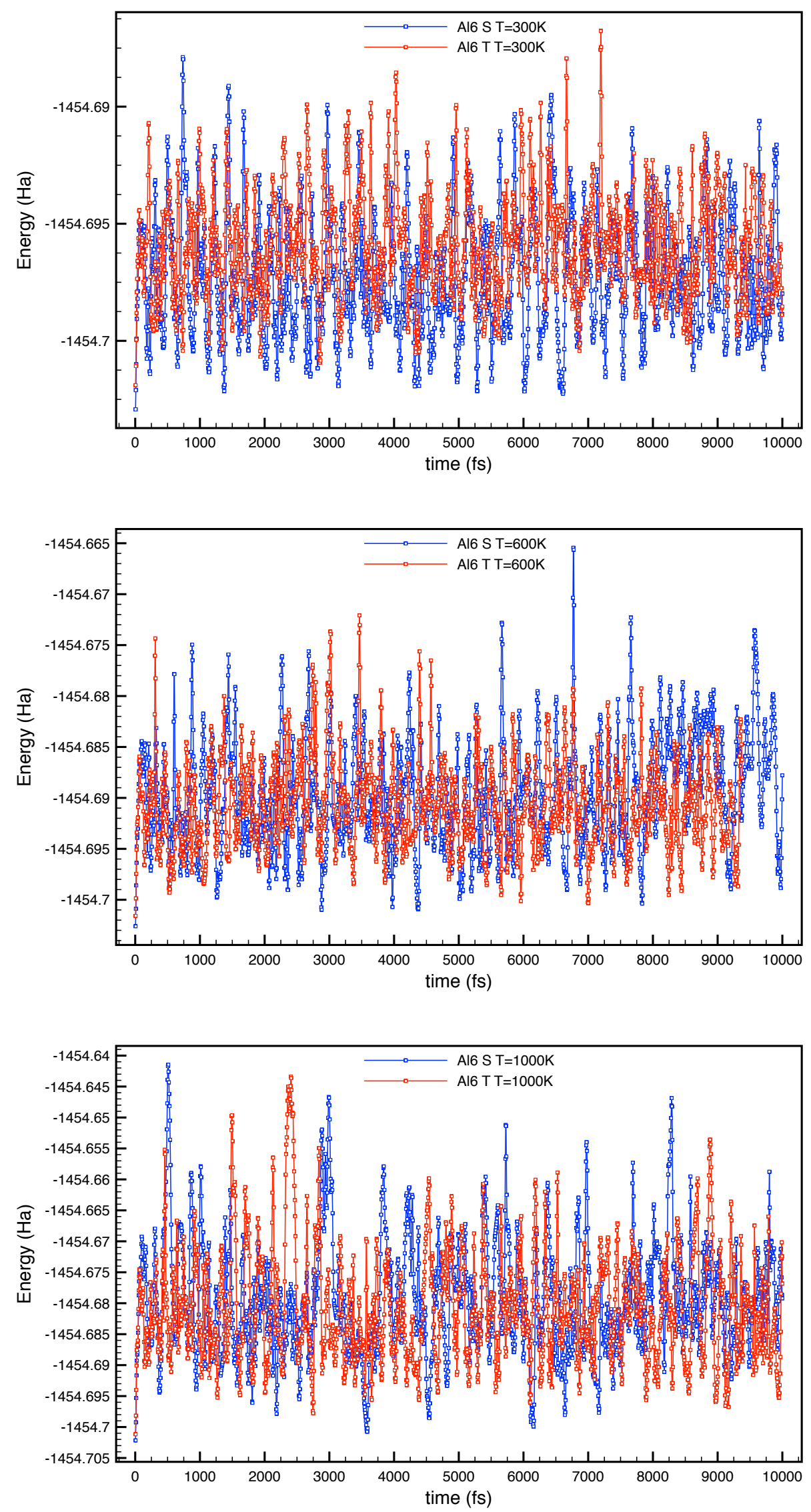

FIG. 1. $\mathrm{Al}_{6}$ Born-Oppenheimer molecular dynamics trajectories computed at TPSS/aug-cc-DVZP level theory at $\mathrm{T}=300,600$ and $1000 \mathrm{~K}$. 

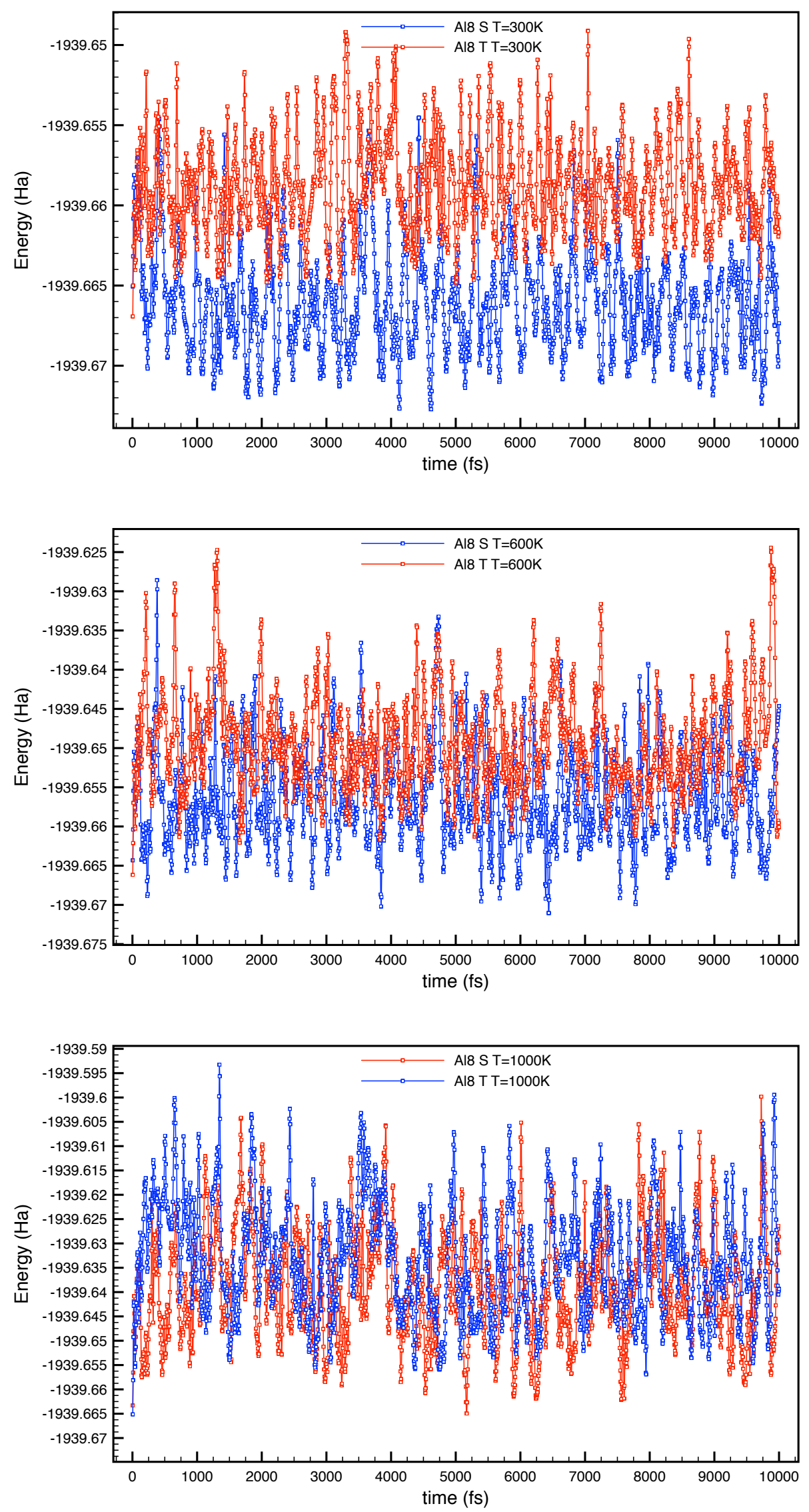

FIG. 2. $\mathrm{Al}_{8}$ Born-Oppenheimer molecular dynamics trajectories computed at TPSS/aug-cc-DVZP level theory at $\mathrm{T}=300,600$ and $1000 \mathrm{~K}$. 


\section{BIBLIOGRAPHY}

1 Z. Fu, G. W. Lemire, G. a. Bishea, and M. D. Morse, The Journal of Chemical Physics 93, 8420 (1990).

${ }^{2}$ P. W. Villalta and D. G. Leopold, The Journal of chemical physics 130, 024303 (2009).

3 D. M. Cox, D. J. Trevor, R. L. Whetten, and A. Kaldor, The Journal of Physical Chemistry 92, 421 (1988). 